\title{
Prevalencia de Síndrome metabólico en pacientes con psoriasis, mayores de $\mathbf{4 0}$ años.
}

\section{RESUMEN}

OBJETIVO: El síndrome metabólico implica aumento en la morbimortalidad por causas cardiovasculares, donde Psoriasis se ha vinculado como un factor de riesgo por su componente inflamatorio crónico endotelial, por lo que este estudio pretende Investigar la prevalencia y el riesgo de desarrollar síndrome metabólico en pacientes con psoriasis; mayores de 40 años en la Consulta Externa de Dermatología del Hospital Escuela.
\end{abstract}

Prevalence of Metabolic Syndrome in patients with Psoriasis, over 40 years. C.E.R ${ }^{1}$ Johanna Melissa Mendoza C ${ }^{2}$. Elmer López L. ${ }^{3}$. María Guadalupe Romero ${ }^{4}$

MATERIAL Y MÉTODOS: El estudio es de carácter descriptivo transversal, el método de obtención de datos fue la encuesta utilizando la técnica de la entrevista estructurada, con preguntas abiertas y cerradas. Se obtuvo una muestra de 40 pacientes con Psoriasis de ambos sexos, de la Consulta Externa de Dermatología, se les investigó criterios de Síndrome Metabólico según ATP III. Los datos fueron ordenados en una matriz y luego tabulados, usando el programa Epi-Info V. 3.5.1 Análisis de los datos: univariado y bivariado. Se aplico las pruebas estadísticas: frecuencias, tendencia central.

RESULTADOS: De los 40 pacientes estudiados con Psoriasis 28(70\%) cumplían criterios para síndrome metabólico, observando una relación de cifras tensionales elevadas en 21 pacientes $(52.5 \%)$, hiperglicemia en 9 pacientes $(22.5 \%)$ y dislipidemia en 20 pacientes (50\%).

CONCLUSIONES: Existe una alta prevalencia de Síndrome Metabólico en los pacientes con Psoriasis por lo que esta debe considerarse un factor de riesgo para desarrollar dicha enfermedad.
PALABRAS CLAVE: Síndrome Metabólico, Psoriasis.

\begin{abstract}
OBJECTIVE: Metabolic syndrome involves increased morbidity and mortality from cardiovascular causes, where Psoriasis has been linked as a risk factor by endothelial chronic inflammatory component, so this study aims to investigate the prevalence and the risk of developing metabolic syndrome in patients with psoriasis; over 40 years in the Dermatology Outpatient Hospital Escuela.

MATERIAL AND METHODS: The study is a descriptive cross-sectional method of data collection was a survey using structured interview technique with open and closed questions. A sample of 40 patients with psoriasis of both sexes from the dermatology outpatients, were investigated criteria for metabolic syndrome according to ATP III. The data were arranged in an array and then tabulated using Epi-Info V. 3.5.1 Data analysis: Univariate and bivariate. Statistical tests were applied: frequencies, central tendency.
\end{abstract}

RESULTS: Of the 40 patients with psoriasis $28(70 \%)$ met criteria for metabolic syndrome, having a relationship of high blood pressure in 21 patients (52.5\%), hyperglycemia in 9 patients $(22.5 \%)$ and dyslipidemia in 20 patients (50\%).

CONCLUSIONS: The high prevalence of metabolic syndrome in patients with psoriasis so this should be considered a risk factor for developing the disease.

KEYWORDS: Metabolic Syndrome, Psoriasis.

\footnotetext{
${ }^{1}$ Comité Editorial de la Revista de Postgrados de Medicina UNAH, Dirección de Docencia e Investigación-HE: Dr. Carlos Vargas Pineda, Dr. Rolando-Aguilera-L, Dra. Guadalupe-Romero-A

2 Residente de 3er. Año Postgrado de Dermatología Universidad Nacional Autónoma de Honduras.

3 Jefe del Post Grado de Dermatología del Hospital Escuela de Tegucigalpa, Honduras.

4 MD, MSP, MASS.
} 
INTRODUCCIÓN: La psoriasis es una enfermedad cutánea crónica, inflamatoria e inmunomediada de etiología desconocida. ${ }^{1} \mathrm{Se}$ ha observado mayor frecuencia de comorbilidades en pacientes con psoriasis que en la población en general lo cual parece estar asociado con esta enfermedad. ${ }^{2}$ Las comorbilidades tradicionalmente asociadas incluyen desordenes inmunomediados, como la artritis psoriatica y la enfermedad intestinal inflamatoria. Sin embargo, cada vez más se ha reconocido la asociación con disfunciones metabólicas, que incluyen la obesidad, el síndrome metabólico (SM) y la enfermedad cardiovascular (ECV), con sus consecuentes efectos sobre la morbimortalidad de los pacientes psoriáticos. ${ }^{3}$ El síndrome metabólico es una combinación de factores de riesgo que incluyen intolerancia a la glucosa, hipertensión, obesidad central ,dislipidemia, y es un fuerte predictor de enfermedad cardiovascular, diabetes y ECV, donde la inflamacion crónica cumple un rol etiológico importante para el desarrollo de estos desordenes, por lo que se ha asociado la psoriasis como factor de riesgo, debido a que es una enfermedad inflamatoria crónica que implica además estrés oxidativo, angiogènesis, y susceptibilidad genética. ${ }^{10} \mathrm{~A}$ nivel mundial se han realizado varios estudios de casos y controles; En Europa se ha informado alta prevalencia en Italia e Israel, con una tasa de $30.1 \%$ y $57.1 \%$ (casos) vs. $20.6 \%$ y $47.4 \%$ (controles). ${ }^{4} \quad$ En Estados Unidos, Philadelphia se identifico el alto riesgo de síndrome metabólico, se obtuvo prevalencia de diabetes $(7.1 \%, 4.4 \%, 3.3 \%)$, hipertensión $(20 \%, \quad 14.7 \%, \quad 11.9 \%)$, hiperlipidemia $(6 \%, 4.7 \%, 3.3 \%)$, obesidad (20.7\%, 15.8\%, 13.2\%) respectivamente. ${ }^{5}$ En Latinoamérica se han hecho múltiples revisiones acerca del tema, en especial en México, Chile y Argentina, sin embargo no se han realizado estudios para comparar 0 determinar la prevalencia del síndrome metabólico en pacientes con psoriasis ${ }^{6}$, al igual en nuestro país hay deficiencias institucionales en el manejo de las estadísticas, no solo de los causantes de esta entidad sino de las condiciones asociadas, por lo que se considero importante la realización de este estudio con el fin 0 el propósito de aumentar el conocimiento de que esta enfermedad junto con el síndrome metabólico aumenta la morbilidad y deteriora su pronóstico, por ende se pretende Investigar la prevalencia y el riesgo de desarrollar síndrome metabólico en pacientes con psoriasis; mayores de 40 años en la Consulta Externa de Dermatología del Hospital Escuela, de Agosto a Septiembre 2009 - 2010. Y mejorar así la calidad de vida de estos pacientes.

\section{MATERIAL Y METODOS}

Se realizo un estudio descriptivo transversal. Efectuado en la Consulta Externa de Dermatologia del Hospital Escuela, en pacientes con psoriasis que asistieron a este servicio, donde se obtuvo una muestra de 40 pacientes, mayores de 40 años de ambos sexos durante 1 un periodo de 1 año de agosto del 2009- 2010; los pacientes fueron seleccionados por muestreo no probabilístico por conveniencia en cuotas, atendidos por

\footnotetext{
${ }^{1}$ Comité Editorial de la Revista de Postgrados de Medicina UNAH, Dirección de Docencia e Investigación-HE: Dr. Carlos Vargas Pineda, Dr. Rolando-Aguilera-L, Dra. Guadalupe-Romero-A

2 Residente de 3er. Año Postgrado de Dermatología Universidad Nacional Autónoma de Honduras.

3 Jefe del Post Grado de Dermatología del Hospital Escuela de Tegucigalpa, Honduras.

4 MD, MSP, MASS.
} 
especialistas y médicos residentes de Dermatologia tomando como criterios de inclusión los siguientes: Población mayor de 40 años con Psoriasis y que quiera participar en el estudio, que no tengan antecedentes de otras enfermedades sistémicas crónicas antes de su diagnóstico y pacientes con Psoriasis que no tengan otra dermatosis aguda $y$ crónica asociada. El método de obtención de datos fue la encuesta utilizando la técnica de la entrevista estructurada, con un instrumento que contenía preguntas abiertas y cerradas, de selección múltiple y literal, distribuidas en 2 secciones: Datos generales y Datos clínicos y laboratoriales.Se realizó el estudio desde el momento en que paciente llego a la Consulta Externa de Dermatologia, en donde se contaba con el instrumento de trabajo previamente elaborado, tomando datos generales, información sociodemográfica como la edad, sexo, lugar de procedencia, ocupación; así como datos clínicos: presión arterial, perímetro abdominal, talla, peso, para medición de índice de masa corporal.

Posteriormente se les solicito que se realizaran en ayuna perfil lipídico (triglicéridos, colesterol total y HDL), glicemia en ayunas, en el laboratorio del Hospital Escuela de acuerdo a los criterios para Síndrome Metabólico por la ATPIII (ver tabla 1). Posteriormente se cito al paciente para revisión de exámenes y seguimiento. Los datos fueron incluidos en Microsoft Excel y analizados en EPI INFO V. 3.5 1. Se obtuvieron medidas de tendencia central, frecuencias, y Chi cuadrado.

Tabla:1 Criterios Diagnósticos de Síndrome Metabólico

\begin{tabular}{|l|l|}
\hline INDICADORES & PUNTUACION \\
\hline $\begin{array}{l}\text { Presión arterial } \\
\text { elevada }\end{array}$ & $>130 / 85$ \\
\hline $\begin{array}{l}\text { Perímetro } \\
\text { abdominal elevado }\end{array}$ & $\begin{array}{l}>102 \mathrm{~cm} \text { en } \hat{\sigma} \\
>88 \mathrm{~cm} \text { en }+\end{array}$ \\
\hline Triglicéridos & $>150 \mathrm{mg} / \mathrm{dl}$ \\
\hline $\begin{array}{l}\text { Colesterol HDL } \\
\text { disminuido }\end{array}$ & $\begin{array}{l}<40 \mathrm{en} \hat{\delta} \\
<50 \mathrm{en} \bigcirc\end{array}$ \\
\hline Glicemia en ayunas & $>110 \mathrm{mg} / \mathrm{dl}$ \\
\hline
\end{tabular}

Fuente : ATPIII (2006)

RESULTADOS: En nuestro estudio se incluyeron 40 pacientes desde Agosto del 2009 a Agosto del 2010, de los cuales 55\% fueron femeninos y $45 \%$ masculino; comprendidos entre los 40 años en adelante.

El rango de edad más frecuente fue entre los 40- 49 años con un $40 \%$, procedentes de todo el país siendo mas frecuente los pertenecientes a Francisco Morazán con $67.5 \%$ y en su mayoría amas de casa $45 \%$.

Del total de pacientes con psoriasis el $70 \%$ (28) cumplió con los criterios según ATP III para el diagnostico de Síndrome Metabólico (ver tabla 2); con predominio del sexo femenino con $45 \%$ (18), en comparación con el sexo masculino con $25 \%$ (10).

Tabla 2: Presencia de Síndrome Metabólico

\begin{tabular}{|l|l|l|}
\hline & & \\
$\begin{array}{l}\text { Presencia de } \\
\text { Síndrome Metabólico }\end{array}$ & Frecuencia & $\%$ \\
\hline No & 12 & 30 \\
\hline Si & 28 & 70 \\
\hline Total & 40 & 100 \\
\hline
\end{tabular}

Fuente: encuesta.

En relación a la edad, de los pacientes con Psoriasis y Síndrome metabólico el $27.5 \%$ (11) tenían entre los 40-49 años.

En cuanto a la ocupación en su mayoría eran amas de casas $37.5 \%(15)$, seguido por comerciantes $12.5 \%$ (5).

\footnotetext{
${ }^{1}$ Comité Editorial de la Revista de Postgrados de Medicina UNAH, Dirección de Docencia e Investigación-HE: Dr. Carlos Vargas Pineda, Dr. Rolando-Aguilera-L, Dra. Guadalupe-Romero-A

2 Residente de 3er. Año Postgrado de Dermatología Universidad Nacional Autónoma de Honduras.

3 Jefe del Post Grado de Dermatología del Hospital Escuela de Tegucigalpa, Honduras.

4 MD, MSP, MASS.
} 
La mayoría de los pacientes con Síndrome Metabólico procedían de Francisco Morazán $50 \%(20)$, y otros de El Paraíso $10 \%$, Comayagua $7.5 \%$ y La Paz 2.5\%.

Se observo que el antecedente de tabaquismo no estaba en la mayoría de los pacientes con Psoriasis y Síndrome Metabólico, ya que solo un 5\% 2/28 tenían este antecedente.

Además se observo que los pacientes con psoriasis y síndrome metabólico en su mayoría tenían alteración del índice de masa corporal con sobrepeso $27.5 \%$ y obesos en igual porcentaje, en comparación con aquellos que no tenían Síndrome Metabólico en los que solo un $2.5 \%$ tenía obesidad, y $17.5 \%$ en sobrepeso; acompañado con niveles bajos de $\mathrm{HDL}$ en $50 \%$ de los pacientes y cifras tensionales elevadas (52.5\%).

En cuanto a los datos clínico laboratoriales se observo lo que está representado en la tabla 3.

\begin{tabular}{|c|c|c|}
\hline Condición & Numero & Porcentaje \\
\hline $\begin{array}{l}\text { Presión Arterial } \\
\text { mayor de } 130 / 85\end{array}$ & 25 & 62.5 \\
\hline $\begin{array}{l}\text { Perímetro } \\
\text { Abdominal } \\
\text { elevado }\end{array}$ & 28 & 70 \\
\hline $\begin{array}{l}\text { Triglicéridos } \\
\text { mayor de } \\
150 \mathrm{mg} / \mathrm{dl}\end{array}$ & 35 & 87.5 \\
\hline $\begin{array}{l}\text { Colesterol HDL } \\
\text { disminuido }\end{array}$ & 22 & 55 \\
\hline $\begin{array}{l}\text { Glicemia en } \\
\text { ayunas mayor de } \\
110 \mathrm{mg} / \mathrm{dl}\end{array}$ & 9 & 22.5 \\
\hline
\end{tabular}

DISCUSION: La Psoriasis es una enfermedad inflamatoria crónica de etiología aun desconocida en el que se han implicado múltiples factores para su inicio y desarrollo. En estudios recientes la han asociado a otras entidades como ECV, enfermedad cardiovascular y endocrinopatías como el Síndrome Metabólico, donde la inflamacion crónica cumple un rol etiológico importante para el desarrollo de estos desordenes, por lo que se ha asociado la psoriasis como factor de riesgo independiente, debido a que es una enfermedad inflamatoria crónica que implica además estrés oxidativo, angiogènesis, y susceptibilidad genética. Dada la importancia de estos últimos datos decidimos realizar este estudio para identificar si nuestros pacientes con psoriasis tienen una mayor prevalencia y un riesgo independiente de desarrollar Síndrome Metabólico, los que nos permitiría mejorar la calidad de vida y el pronóstico de estos pacientes; por lo que en nuestro estudio se incluyeron pacientes mayores de 40 años de edad, ya que es en este rango de edad que prevalece el Síndrome Metabólico en la población en General. Del total de los 40 pacientes que fue nuestra muestra el $70 \%$ presenta Síndrome Metabólico, datos que son comparables con otros países, en donde se han realizado varios estudios de casos y controles que demuestran que los pacientes con Psoriasis tienen una mayor prevalencia del Síndrome Metabólico, Europa (57.1\%), y EEUU. con $63 \%$.

La Presión arterial elevada y la diabetes por si solas han sido asociadas de forma independiente con tasas de $20 \%$ y $7.1 \%$ respectivamente, en nuestros pacientes con psoriasis con o sin Síndrome Metabólico el $62.5 \%$ presentaron cifras tensionales elevadas, $22.5 \%$ niveles de glicemia elevados y el $27.5 \%$ tienen sobrepeso, último dato que se asemeja a la de los EEUU. con $20.7 \%$. La presencia de índice de masa corporal mayor de 25(sobrepeso) fue uno de los factores que más influyeron para la identificación de síndrome metabólico en los sujetos del estudio, tal como es demostrado en estudios internacionales y/o lo reportado por la ATP

\footnotetext{
${ }^{1}$ Comité Editorial de la Revista de Postgrados de Medicina UNAH, Dirección de Docencia e Investigación-HE: Dr. Carlos Vargas Pineda, Dr. Rolando-Aguilera-L, Dra. Guadalupe-Romero-A

2 Residente de 3er. Año Postgrado de Dermatología Universidad Nacional Autónoma de Honduras.

3 Jefe del Post Grado de Dermatología del Hospital Escuela de Tegucigalpa, Honduras.

4 MD, MSP, MASS.
} 
$1 \mathrm{II}^{9}$, pero considero importante mencionar que en el estudio se identifico la presencia de síndrome metabólico en sujetos con peso normal, lo que nos dice que no todo paciente con síndrome metabólico es obeso y que en estos pacientes la presencia de otro factor de riesgo como ser la hipertrigliceridemia, disminución del colesterol HDL contribuye al diagnostico de SM, por tanto se sugiere la identicaciòn del constitucional en este tipo de pacientes.

CONCLUSION: La prevalencia del síndrome metabólico en la población adulta con Psoriasis estudiada corresponde a un $70 \%$, por lo que la Psoriasis se debe considerar un factor de riesgo para desarrollar dicha enfermedad. La presencia de Síndrome Metabólico predomino en el sexo femenino $(55 \%)$, en pacientes con sobrepeso $(27.5 \%)$, entre los 40 - 49 años de edad (40\%).

Por lo anterior se debería protocolizar la búsqueda de Síndrome Metabólico en los pacientes con psoriasis que asisten a la Consulta Externa de Dermatologia del Hospital Escuela, debido a que la presencia de esta entidad acarrea un elevado riesgo potencial de complicaciones cardiovasculares futuras, por lo que sería oportuno la intervención o abordaje multidisciplinario de estos pacientes para así mejorar la calidad de vida de estos, y disminuir la morbimortalidad debido a que el Síndrome Metabólico se ha asociado como factor de riesgo para Infarto Agudo del Miocardio, ECV y arterioesclerosis.

\section{BIBLIOGRAFÍA}

1. Griffiths, Barker Jwn. Pathogenesis and clinical features of psoriasis. Lancet 2007; 370:263-71.

2. Christopher E. Comorbidities in psoriasis. Clinics Dermatology 2007; 25(6):529-34.

3. Claudia de la Cruz F., Consuelo C. Psoriasis y sus comorbilidades sistémicas. Rev. Chilena Dermatol: 2008; 24(4):348-351.

4. P. Gisondi, G. Tessari, A. Conti, S. Piaserico, S. Schianchi, A. Peserico, A. Giannetti, G. Girolomoni. Prevalence of metabolic syndrome in patients with psoriasis: a hospital-based case-control study British Journal of Dermatology: 2007; 157, 68-73.

5. Andrea N, Daniel S, Xingmei W, David M, Andrea $T$, Joel G. Prevalence of cardiovascular risk factors in patients with psoriasis. J Am Acad Dermatol: 2006; November, 829 -835.

6. Poletti E, Sandoval M, Esperon G. Psoriasis: ¿Espejo de riesgo cardiovascular? .Med Int Mex: 2008; 24(3): 210-6.

7. Israel A, María Antonia G, Olga T, Leopoldina F, Lincheta, Margarita P. psoriasis vulgar, estudio descriptivo de 200 pacientes: Rev. Cubana Med 2002; 41(1):12-5.

8. Srjdan $P$, Robert $S$, Jeffrey K, Fangchao M, Lisa M, Daniel G, Federman. Association of Psoriasis with Coronary Artery, Cerebrovascular, and Peripheral Vascular Diseases and Mortality. Arch Dermatol: 2009; 145 (6):700-703.

9. Alice B. Gottlieb, Frank D. Comorbidities in Patients with Psoriasis. The American Journal of Medicine: 2009; $122,12$.

10. Arnon D, Harel G, Yaakov H, Doron Z, Jonathan, Dan Y, Daniel A.V. Psoriasis and the Metabolic Syndrome. Acta Derm Venereol: 2007; 87: 506509

11. Pineda CA. Síndrome Metabólico: definición, historia, criterios. Colomb Med: 2008; 39: 96-106.

12. Darwin DEEN. Metabolic Syndrome: Time for Action. American Family Physician: 2004; 69 (12): 2875- 2882.

13. Jorge Barrientos, Cecilia Varela. Síndrome Metabólico en pacientes diabéticos e hipertensos en la consulta Externa de Medicina Interna. Revista Médica de los Postgrados de Medicina UNAH: 2007; 10 (3).

14. Shai A, Vardy D, Zvulunov. Psoriasis, biblical afflictions and patients dignity: 2002; 141(5): 479482, 496 .

15. Glickman. A note on the history of psoriasis. Irish Journal of Medical Science: 1995; 30(3): 141 - 142.

16. Wolff, Goldsmith, Katz, Gilchrest, Paller, Leffell. Fitzpatrick's Dermatology in General Medicine 7 a ed. 2008; 4: 169- 193.

17. Johann E. Gudjonsson, James T. Elder. Psoriasis: Epidemiology. Clinics in Dermatology: 2007; 25: 535-546.

18. International psoriasis council. Revisión del IPC Sobre la Psoriasis: 2009; 5: 2.

19. Menna S, Tania S, Carlos O. Factores de Riesgo de Pacientes con Psoriasis en la Consulta Externa, Dermatología, Hospital Escuela. Rev. Fac. Cienc. Méd: 2008; enero-junio.

20. Stephen K, Joel M. Update on the natural history and systemic treatment of psoriasis. Adv. Dermatology: 2008; 24: 171-196.

21. P.M. Lowe, .J. Jackson, Cooper, L.Schrieber. The endothelium in psoriasis. British Journal of Dermatology: 1995; 132: 497-505.

\footnotetext{
${ }^{1}$ Comité Editorial de la Revista de Postgrados de Medicina UNAH, Dirección de Docencia e Investigación-HE: Dr. Carlos Vargas Pineda, Dr. Rolando-Aguilera-L, Dra. Guadalupe-Romero-A

2 Residente de 3er. Año Postgrado de Dermatología Universidad Nacional Autónoma de Honduras.

3 Jefe del Post Grado de Dermatología del Hospital Escuela de Tegucigalpa, Honduras.

4 MD, MSP, MASS.
} 


\section{Revista de los Postgrados de Medicina UNAH \\ Vol. $13 \mathrm{~N}^{\circ} 3$ Agosto-Octubre 2010}

22. Dorothea M, Stefan J, Michael S. Enno C, Michael $W$. Increased prevalence of the metabolic syndrome in patients with moderate to severe psoriasis. Arch Dermatol Res: 2006; 298:321-328.

23. Jonathan S, Arnon D, Michael D, Emilia H, Gabi C, Anna V, Eyal K, Anthony $\mathrm{H}$. The association between psoriasis, diabetes mellitus, and atherosclerosis in Israel: A case-control study. J. Am Acad Dermatol: 2007; April, 629-634.

24. Yi-Ju Chen, Chun-Ying Wu, Jui-Lung Shen, SzuYing Chu, Chih-Kang Chen, Yun-Ting Chang, Chuan-Mu Chen. Psoriasis Independently Associated with Hyperleptinemia Contributing to Metabolic Syndrome. Arch Dermatol: 2008; 144 (12):1571-1575

25. Ludewig C, Rodríguez A, Zambrano A. Taller de metodología de la investigación: Universo y muestra. Barquisimeto: 1998

26. Martin Robledo J. Diseño de muestreo. Nure Investigation: 2005; 12.

27. Hurtado J. Verbos en la investigación. Sypal: 1995.Wayne W. Daniel. Bioestadística. Base para el análisis de las ciencias de salud 3ed.1996; 4.

${ }^{1}$ Comité Editorial de la Revista de Postgrados de Medicina UNAH, Dirección de Docencia e Investigación-HE: Dr. Carlos Vargas Pineda, Dr. Rolando-Aguilera-L, Dra. Guadalupe-Romero-A

2 Residente de 3er. Año Postgrado de Dermatología Universidad Nacional Autónoma de Honduras.

3 Jefe del Post Grado de Dermatología del Hospital Escuela de Tegucigalpa, Honduras.

4 MD, MSP, MASS. 